\title{
Endometrial thickness following early miscarriage in IVF patients - is there a preferred management approach?
}

\author{
Gilad Karavani ${ }^{1 \dagger} \mathbb{B}$, Heli Alexandroni ${ }^{2 \dagger}$, Daniel Sheinin ${ }^{3}$, Uri P. Dior ${ }^{1}$, Alex Simon$^{1}$, Assaf Ben-Meir ${ }^{1}$ and
} Benjamin Reubinoff ${ }^{*}$

\begin{abstract}
Background: Endometrial thickness (ET) has previously been shown to positively correlate with implantation and clinical pregnancy rates. Pregnancies achieved using in-vitro fertilization (IVF) technique are prone to higher rates of early miscarriage. The aim of this study was to compare the effects of expectant management, medical treatment (Misoprostol) and dilation and curettage (D\&C) for early miscarriage following IVF cycles on the subsequent cycle outcomes - endometrial thickness and reproductive outcomes.

Methods: A retrospective cohort study of women who underwent embryo transfer, conceived and had first trimester miscarriage with at least one subsequent embryo transfer. ET measurements during fresh or frozenthawed IVF cycles were assessed for each patient. Comparisons of ET differences between the miscarriage and the subsequent cycles, as well as reproductive outcomes, were performed according to the initial miscarriage management approach.

Results: A total of 223 women were included in the study. Seventy-eight women were managed conservatively, 61 were treated with Misoprostol and 84 women underwent D\&C. Management by D\&C, compared to conservative management and Misoprostol treatment was associated with higher prevalence of a significant $(>2 \mathrm{~mm}) \mathrm{ET}$ decrease $(29.8 \% \%$ vs. 14.1 and $6.6 \%$, respectively; $p<.001)$ and was the only approach associated with a significant increase in the rates of ET under 7 and $8 \mathrm{~mm}$ in the following cycle ( $p=0.006$ and 0.035 ; respectively). Clinical pregnancy rates were significantly lower following D\&C compared with conservative management and Misoprostol (16.7\% vs. 38.5 and $27.9 \%$, respectively; $p=0.008)$ as well as implantation rate $(11.1 \%$ vs. $30.5 . \%$ and 17.7 , respectively; $p<0.001)$.

Conclusion: Our data suggest that D\&C management of a miscarriage is associated with decreased ET and higher rates of thin endometrium in the subsequent IVF cycle, compared with conservative management and Misoprostol treatment. In addition, implantation and pregnancy rates were significantly lower after D\&C.
\end{abstract}

Keywords: Endometrial thickness, Miscarriage, Expectant management, Misoprostol, Dilation and curettage

\footnotetext{
* Correspondence: BenR@hadassah.org.il

${ }^{\dagger}$ Gilad Karavani and Heli Alexandroni contributed equally to this work

${ }^{1}$ Infertility and IVF Unit, Department of Obstetrics and Gynecology, Hadassah

Ein-Kerem Medical Center and Faculty of Medicine, Hebrew University of

Jerusalem, Jerusalem, Israel

Full list of author information is available at the end of the article
}

(c) The Author(s). 2021 Open Access This article is licensed under a Creative Commons Attribution 4.0 International License, which permits use, sharing, adaptation, distribution and reproduction in any medium or format, as long as you give appropriate credit to the original author(s) and the source, provide a link to the Creative Commons licence, and indicate if changes were made. The images or other third party material in this article are included in the article's Creative Commons licence, unless indicated otherwise in a credit line to the material. If material is not included in the article's Creative Commons licence and your intended use is not permitted by statutory regulation or exceeds the permitted use, you will need to obtain permission directly from the copyright holder. To view a copy of this licence, visit http://creativecommons.org/licenses/by/4.0/ The Creative Commons Public Domain Dedication waiver (http://creativecommons.org/publicdomain/zero/1.0/) applies to the data made available in this article, unless otherwise stated in a credit line to the data. 


\section{Introduction}

The success rate of an In-vitro fertilization (IVF) treatment depends on multiple factors. Among them are patient's age, number and quality of transferred embryos, type of stimulation protocol and endometrial characteristics [1]. Features of the endometrium, evaluated with vaginal ultrasound (US), include endometrial thickness (ET), pattern, volume and blood flow [2].

The correlation between ET and implantation rate has been widely studied in the literature with inconsistent results. It is still uncertain whether there is a minimal ET threshold that is essential for successful implantation. Some studies concluded that a thin endometrium less than $7 \mathrm{~mm}(\mathrm{~mm})$ is associated with a greater risk for unfavorable outcome of IVF treatments [3-9]. Others reported a decreased fecundity rate for endometrium thickness below $8-10 \mathrm{~mm}[10-14]$. A thick endometrium has also been suggested to be associated with implantation failure. Weissman et al., reported reduced implantation and pregnancy rates in a group of patients with ET above $14 \mathrm{~mm}$, compared to 7-14 mm [15]. Nonetheless, case reports showed that IVF pregnancies can be carried out even with a minimal ET of $4 \mathrm{~mm}$ [16] or an increased thickness, up to $20 \mathrm{~mm}$ [17]. Regardless of the inconclusive literature regarding the optimal ET, most studies agree that within the low range of ET there is a positive correlation between ET and implantation rate, particularly with pregnancies achieved by assisted reproductive technology (ART) $[1,15]$.

Pregnancies that are conceived via IVF are suggested to carry a slightly increased risk of miscarriage, compared with spontaneous conceptions [18]. Management of miscarriage include either expectant management, medical treatment (antiprogesterone or most commonly a prostaglandin analogue) or surgical uterine curettage known as dilation and curettage (D\&C).

Due to a paucity of evidence regarding the effect of miscarriage management approach on subsequent ET and pregnancy outcomes, the dilemma which management approach is preferred remains unsolved. In this study, we sought to evaluate the effects of the different miscarriage management approaches on ET, clinical pregnancy and delivery rates in the subsequent IVF cycle. This study aims to provide data that may assist physicians when counseling patients with early miscarriage regarding the preferred management option.

\section{Materials and methods}

\section{Study population}

This retrospective cohort study included women that presented with an early miscarriage (first trimester) following a fresh or frozen-thawed (FT) embryo transfer in IVF cycles between 2006 and 2017.
Diagnosis of early miscarriage was made by vaginal US scan during a routine first trimester examination or following symptoms of abdominal contractions or vaginal bleeding. Patients with a miscarriage under 12 weeks (calculated according to the embryo transfer date) were included in the study. According to the management policy in our institution, each patient was fully informed and consulted about three miscarriage management possibilities: expectant management, medical treatment (Misoprostol) and dilation and curettage (D\&C) followed by a specific consent form given and signed by the patients who chose medical or surgical approaches according to individual preferences.

The patients were divided into three management groups that were defined according to the initial miscarriage management approach that was selected by the patients.

The first group included patients who preferred expectant management and had undergone complete abortion by the time of follow-up visit, 2 weeks after the initial diagnosis. In cases of lack or incomplete spontaneous expulsion of pregnancy products from the uterine cavity within 2 weeks, medical or surgical interventions were performed according to patients' choice. Such cases were not included in the study.

The second group included patients who chose medical treatment management. They received four $200 \mathrm{mcg}$ Misoprostol tablets $(800 \mathrm{mcg}$ in total), inserted into the posterior fornix of the vagina. After 48 -h, a second dose of $800 \mathrm{mcg}$ vaginal Misoprostol was given if US scan demonstrated a remaining gestational sac or thick endometrium (above $25 \mathrm{~mm}$ ). Two weeks after the administration of Misoprostol, treatment was considered successful if an empty uterine cavity was demonstrated by a transvaginal US. Patients with successful medical management were included in the second group, while patients with retained products of conception who underwent further evacuation by $D \& C$ were not included in the study.

The third group included patients who preferred surgical management. They underwent, under general anesthesia, dilation of the cervix, vacuum aspiration of gestational products, followed by sharp curettage (D\&C) to complete evacuation and confirm that the uterine cavity was empty. D\&Cs were routinely performed by an attending physician, besides for cases that required an urgent procedure during on calls. In these cases, the D\&Cs were performed by residents. Guidance of curettage by US was not routinely performed.

Exclusion criteria were miscarriages before 6 or beyond 12 weeks of gestation, chemical pregnancies (no evidence of gestational sac) and pregnancies suspected to be ectopic. Patients which experienced complications of miscarriage managements including excessive uterine 
bleeding (resulting in a need for blood transfusion), perforation of the uterus, fever and a need for operative hysteroscopy for the removal of retained products of conception were excluded. As mentioned above, patients with failed conservative or Misoprostol treatments that eventually underwent $\mathrm{D} \& \mathrm{C}$ procedure and for whom the initial and the final management approaches were different, were not included in the study to prevent a selection bias that may result in inferiority of the D\&C group's outcomes.

General and gynecological medical history was collected for all the patients. For each patient, at least two IVF cycles within 18 months were evaluated: the miscarriage cycle and the subsequent cycle. When the type of embryo transfer (fresh or frozen-thawed (FT)) in the miscarriage and subsequent cycles was different, the first additional subsequent cycle with a similar type of embryo transfer as the miscarriage cycle was also evaluated.

\section{Endometrial thickness evaluation}

During each IVF cycle, ET was routinely measured when patients underwent US examination. We collected data regarding measurements at three points of each IVF cycle: at day three of menstrual period, at the day of hCG administration (fresh IVF cycles) or LH surge (natural frozen-thawed cycles) or first day of progesterone administration (estrogen and progesterone frozenthawed cycles) (hCG/LH/P), and at "mid-cycle" between the 7th and 10th day of menstrual period). ET was defined as the maximal distance between the echogenic interfaces of the myometrium and the endometrium in a midsagittal plane of the uterus. Ultrasound examinations at day three were performed either at the community services or at our IVF unit. All ultrasound measurements at hCG/LH/P administration were performed at our IVF unit by a single US specialist in the same setting. These measurements were done by transvaginal US using a Voluson $^{\mathrm{Tx}}$ GE E6 system device and IC 5-9-D $\mathrm{MHz}$ transvaginal probe (GE Healthcare, Milwaukee, WI).

\section{IVF protocols and embryo transfer}

Controlled ovarian stimulation included Gonadotropinreleasing hormone $(\mathrm{GnRH})$ using either long or short agonist protocols or an antagonist protocol. Oocyte retrieval was performed under transvaginal ultrasound guidance and general anesthesia. Intracytoplasmic sperm injection (ICSI) was used in all cases of male factor infertility and in cases of unexplained infertility with previous low fertilization rates. Embryo quality was determined by cell number, symmetry and fragmentation and was graded "A-C". Embryos were cultured in a one-step medium ("SAGE 1-step" (SAGE, Al-rad medical, Nes Ziona, Israel) at $37^{\circ} \mathrm{C}, 5.7 \% \mathrm{CO} 2$, and $5 \% \mathrm{O} 2$ (Unisense FertiliTech, Aarhus, Denmark). At the time period of the study, transfer of embryos at cleavage stage was practiced in vast majority of cases $(97 \%)$ in our unit. The number of embryos that were transferred was 1-3 embryos, in line with national fertility society guidelines. Two catheters were optional for embryo transfer (upon operator preference) - the Edwards-Wallace catheter (Classic Embryo Replacement Catheter; Smiths Medical, Hythe, Kent, U.K.) or the SIVF catheter (K-Jets-7019SIVF; Cook IVF, Eight Miles Plains, Queensland, Australia). Frozen thawed embryo transfers were performed in natural cycles or artificial cycles using exogenous estradiol (2 mg Q8h) and vaginal progesterone supplementation (Utrogestan; Besins healthcare, Paris, France $400 \mathrm{mg}$ Q12h).

\section{Outcomes}

The primary outcome was alterations in ET following the three management approaches of miscarriage. ET assessed at the day of hCG/ LH/P during the primary miscarriage cycle was compared to both the subsequent and the first subsequent cycles with the same type of embryo transfer (fresh or thawed). This parameter was evaluated as a continuous as well as categorical (ET decrease of more than $2 \mathrm{~mm}$ and ET thinner than 6,7 and $8 \mathrm{~mm}$ ) parameter. Secondary outcomes included implantation, clinical pregnancy, miscarriage and live birth rates in the cycles that followed the miscarriage, comparing the three management approaches. Additional parameters assessed were infertility cause, type of IVF cycle and protocol, the baseline FSH $(\mathrm{mIU} / \mathrm{ml})$, the peak estradiol level ( $\mathrm{pmol} / \mathrm{l})$, the total FSH given and the ET threshold for implantation. In fresh embryo transfer cycles, data regarding number of aspirated and mature oocytes, grade of embryos, quality and number of embryos transferred or frozen were also evaluated.

\section{Ethical approval}

This study was approved by the Human Investigation Review Board of Hadassah Hebrew University Medical center (IRB 0657-17-HMO) and conforms to the provisions of the declaration of Helsinki.

\section{Statistical analysis}

The association between categorical variables was tested using the Pearson $\chi^{2}$ test, as well as the Fisher's exact test, as indicated. For quantitative variables, the comparison between independent variables of the three study groups in both the miscarriage cycle and the first cycle following the miscarriage was performed using the oneway analysis of variance (ANOVA) followed by the PostHoc test for multiple comparisons with the additional Kruskal-Wallis (KW) test and reported it in parameters with unequal distribution. 
KW test was also used when assessing the differences between groups in percentage of change in ET between cycles for each group. Post-hoc Scheffe test was used to identify the coupled groups differences for equal variance such as differences between cycles of endometrial thickness (mm). Post hoc Bonferroni test was performed using the Non-parametric approach for assessment of change in ET ( $\mathrm{mm}$ and \%) between the study groups.

Changes in rates of ET thinning below the cutoffs of 6 , $7,8 \mathrm{~mm}$ between cycles in each group was tested by the McNemar Test.

The logistic regression univariate and multivariate models were applied to assess the effect of several variables on a dichotomous dependent variable. All tests applied were two-tailed, with a $p$-value of $P<0.05$ considered statistically significant.

\section{Results}

\section{Study population and miscarriage cycle data}

Between 2006 and 2017 there were 327 couples at our IVF unit, which conceived and had first trimester miscarriage with at least one subsequent embryo transfer cycle in our unit. Ninety-six couples were excluded due to missing data regarding the miscarriage cycle or the subsequent cycle. The data required for the study was available for 231 couples. Eighty couples were conservatively managed, 67 were treated with Misoprostol and 84 underwent D\&C. Allocation to one of the three management groups was determined according to the initial treatment approach chosen by the patients. Of the patients who received Misoprostol treatment, D\&C was later performed in six patients due to incomplete expulsion of conception products and among the patients who chose expectant management, $D \& C$ was later performed in two patients due to incomplete expulsion of conception products. These patients were excluded from the analysis. Accordingly, in our analysis the conservative management group finally included 78 patients, the Misoprostol group included 61 patients and the D\&C group included 84 patients.

D\&C procedure data were available for $72.4 \%(63 / 87)$ of women. An attending performed the $\mathrm{D} \& \mathrm{C}$ in $85.7 \%$ $(54 / 63)$ of women and US was used only in a few procedures $(4 / 63)$. Vacuum curettage was performed in nearly all cases $(93.6 \%(59 / 63))$, followed by a sharp curettage in all cases. The ET in the following IVF cycle of patients after D\&C by an attending physician (mean ET of $9.3 \mathrm{~mm}$ with an ET decrease of $1.1 \mathrm{~mm}$ between miscarriage and subsequent IVF cycles and only 3 out 54 patients with an endometrium thinner than $6 \mathrm{~mm}$ ) or by a resident (mean ET of $8.4 \mathrm{~mm}$ with an ET decrease of $1.0 \mathrm{~mm}$ between miscarriage and subsequent IVF cycles and only 1 out of 9 patients with an endometrium thinner than $6 \mathrm{~mm}$ ) was not significantly different.
The characteristics of the patients (Table 1) did not significantly differ between the groups, except for the rate of past deliveries (assessed by the Kruskal-Wallis test) which was lower in the conservative management compared to the $\mathrm{D} \& \mathrm{C}$ group. The groups did not differ with regard to the use of any medication, previous gynecologic operations, anatomical uterine abnormalities, previous ectopic pregnancy, rate of patients with previous miscarriages and previous $\mathrm{D} \& \mathrm{C}$ (the remaining cases were managed by expectant management or by Misoprostol), ovulation induction protocol (agonist or antagonist) and cause of infertility. The most prevalent causes of infertility were male factor (total 39.6\%) and unexplained infertility (27.3\%). Ovulation-related or mechanical factor were the cause of infertility in $22 \%$ of patients, and preimplantation genetic diagnosis (PGD) procedure was indicated in about $4 \%$ of them.

A significant difference was noted in the gestational week at miscarriage diagnosis $(p=0.04)$ between all three management groups (by post hoc Dunnett test), ranging from $6.7 \pm 1.3$ weeks in the conservative management group to $8.6 \pm 1.6$ weeks in the $\mathrm{D} \& \mathrm{C}$ treatment group (Table 1).

The three management groups had similar proportions of fresh or frozen-thawed embryo transfers in the subsequent cycle, as well as similar time interval between cycles, peak Estradiol levels and embryo quality (presented as mean rates of embryos graded A-C) (Table 2).

\section{The effect of miscarriage management on endometrial thickness in the subsequent cycle}

Analysis of ET at miscarriage and subsequent cycles, at the time of hCG administration in fresh cycles or LH surge /progesterone administration in frozen-thawed cycles $(\mathrm{hCG} / \mathrm{LH} / \mathrm{P})$, revealed significant differences between the miscarriage management approaches (Table 2). $D \& C$ was associated with a greater average reduction in ET per patient at $\mathrm{hCG} / \mathrm{LH} / \mathrm{P}$ day in the subsequent cycle compared to conservative management and Misoprostol $(-1.0 \mathrm{~mm}$ vs. $0.0 \mathrm{~mm}$ and $0.2 \mathrm{~mm}$, respectively; $p=0.001)$. When analyzing the overall mean difference in ET in the miscarriage and the subsequent cycle for each group separately, there was a significant reduction in ET in the D\&C group $(1.0 \mathrm{~mm}$ decrease in ET, $p=$ 0.002 ) while the mean ET was not significantly altered in both conservative management $(p=0.99)$ and Misoprostol $(p=0.60)$ groups.

In accordance with this finding, the rates of a significant ET decrease (more than $2 \mathrm{~mm}$ ) at hCG/LH/P day between the cycles was significantly higher following D\&C compared to the conservative and Misoprostol methods (29.8\% vs. 14.1 and $6.6 \%$, respectively; $p<0.001$, assessed by post hoc Bonferroni test. Furthermore, tested by the McNemar test, management by $\mathrm{D} \& \mathrm{C}$ was 
Table 1 Characteristics of the patients and the IVF cycle that gave rise to miscarriage conception in the different management groups

\begin{tabular}{|c|c|c|c|c|}
\hline Parameter & Conservative management & Misoprostol & $D \& C$ & $P$ value \\
\hline $\mathrm{N}$ of patients & 78 & 61 & 84 & \\
\hline Age (years) & $34.6 \pm 6.6(35(29-41))$ & $37.1 \pm 5.0(39(33-41))$ & $35.7 \pm 5.6(37(31-40))$ & 0.06 \\
\hline BMI & $25.8 \pm 2.8(25(23-28))$ & $24.3 \pm 6.1(23(19-30))$ & $26.7 \pm 7.8(24(21-30))$ & 0.61 \\
\hline \multicolumn{5}{|l|}{ Obstetric background } \\
\hline Gravidity & $1.3 \pm 1.5(1(0-2))$ & $1.8 \pm 2.2(1(0-3))$ & $1.8 \pm 1.8(2(2-3))$ & 0.16 \\
\hline Past deliveries & 28/72 (38.9\%) & $27 / 59(45.8 \%)$ & 49/81 (59.3\%) & 0.04 \\
\hline Prior miscarriages & $0.8 \pm 1.2(0(0-1))$ & $1.1 \pm 1.8(1(0-2))$ & $0.9 \pm 1.4(0(0-1))$ & 0.41 \\
\hline Patients with a previous $D \& C$ & $17.9 \%$ & $25.5 \%$ & $19.7 \%$ & 0.83 \\
\hline Infertility (years) & $4.1 \pm 4.8(3(1-5))$ & $2.6 \pm 1.8(2(1-4))$ & $4.2 \pm 3.3(3(2-6))$ & 0.08 \\
\hline Day 3 FSH levels ${ }^{a}$ (mIU/ml) & $7.7 \pm 6.9(6(5-9))$ & $8.4 \pm 7.5(7(6-9))$ & $6.9 \pm 3.1(7(5-8))$ & 0.41 \\
\hline \multicolumn{5}{|l|}{ Miscarriage cycle } \\
\hline $\begin{array}{l}\text { Peak E2 levels (Fresh cycles, } \\
\text { pmol/l) }\end{array}$ & $\begin{array}{l}6146.2 \pm 3939.9(5477(2896- \\
8593))\end{array}$ & $\begin{array}{l}5910.9 \pm 3464.5(4942(3928- \\
7866)\end{array}$ & $\begin{array}{l}6267.3 .5 \pm 4273.0(5654(3547- \\
8104))\end{array}$ & 0.53 \\
\hline Endometrium $(\mathrm{mm})$ at hCG/LH/P & $9.9 \pm 1.8(10(9-12))$ & $10.1 \pm 2.0(10(9-11))$ & $10.1 \pm 2.1(10(9-12))$ & 0.81 \\
\hline$>1$ embryonal sac & $8(10.5 \%)$ & $3(4.9 \%)$ & $7(7.7 \%)$ & 0.48 \\
\hline Week of miscarriage & $6.7 \pm 1.3(6(6-7))$ & $7.7 \pm 1.3(7(7-9))$ & $8.6 \pm 1.6(8(8-10))$ & $\begin{array}{l}< \\
0.001^{b}\end{array}$ \\
\hline $\begin{array}{l}\text { Hysteroscopy for retained } \\
\text { placenta }\end{array}$ & $3(4.2 \%)$ & $1(1.8 \%)$ & 0 & 0.13 \\
\hline
\end{tabular}

Data presented as mean \pm SD (Median (IQR)) or $n(\%)$

Statistical analysis was performed using the Pearson Chi-Square test or Fisher's Exact Test as indicated for categorical data and ANOVA or Kruskal-Wallis test (for variables with unequal distribution) for continuous parameters

Note: BMI Body mass index, D\&C Dilation and curettage, FSH Follicular stimulating hormone, $h C G / L H / P$ Day of human chorionic gonadotropin administration in

fresh IVF cycles, luteinizing hormone surge or progesterone administration in frozen-thawed cycles

${ }^{a}$ Baseline FSH levels measured in hormone panel test at day three of menstrual period (prior to initiation of hormonal therapy)

${ }^{b}$ Post hoc Dunnett T3 test showed significant difference between all the study groups

the only approach associated with a significant increase in the rates of ET thinning under 7 and $8 \mathrm{~mm}$ in the following cycle ( $p=0.006$ and 0.035 ; respectively), while an increase in the prevalence of patients with thinning of the endometrium to below 7 and $8 \mathrm{~mm}$ was not demonstrated following conservative management or Misoprostol treatment. ET thinning below $6 \mathrm{~mm}$, though showing similar trend in the D\&C group, occurred in only few cases and did not reach statistical significance.

Univariate analyses, performed to examine the association between parameters of the miscarriage cycle and ET above $8 \mathrm{~mm}$ in the subsequent cycle, identified these factors: previous delivery, miscarriage management approach and ET at hCG/LH/P in the miscarriage cycle, both as a continuous parameter and as a categorical parameter $(>8 \mathrm{~mm})$. In a multivariable logistic regression analysis, all of these 3 parameters were independently associated with endometrium thickness above $8 \mathrm{~mm}$ at hCG/LH/P in the following cycle (Table 3).

To analyze possible biases that may affect ET (as a continuous parameter) at the subsequent cycle, the potential correlation between various patients' parameters (basic characteristics including age, previous gynecologic operations, anatomical uterine abnormalities, previous
Cesarean sections (CS), D\&C or Misoprostol treatment, week of gestation, IVF protocol and fresh or frozen embryo transfer) and ET was evaluated. There was no correlation between any of the parameters and ET, including patients' age and week of miscarriage. Linear modeling (ANCOVA) demonstrated that the only parameters significantly associated with ET at the subsequent cycle were the miscarriage management approach and $\mathrm{ET}$ at hCG/progesterone administration day in the miscarriage cycle ( $p<0.05$ for both).

\section{The effect of miscarriage management on subsequent cycle outcomes}

Reproductive outcomes were analyzed in the three study groups to complement the ET analysis (Table 2). Implantation rate was significantly lower in the subsequent IVF cycle following D\&C compared to conservative and Misoprostol management groups $(11.1 \%$ vs. 30.5 and $17.7 \%$, respectively; $p<0.001$ ).

Additionally, clinical pregnancy rates were also significantly lower in the subsequent IVF cycle following D\&C compared to conservative and Misoprostol managements $(16.7 \%$ vs. 38.5 and $27.9 \%$, respectively, $p=0.008)$. The main difference for both parameters was derived 
Table 2 Basic parameters and endometrium thickness (ET) in the cycle consecutive to the miscarriage cycle of the different management groups

\begin{tabular}{|c|c|c|c|c|}
\hline Parameter & Conservative management & Misoprostol & $D \& C$ & $\begin{array}{l}P \\
\text { value }\end{array}$ \\
\hline $\mathrm{N}$ of patients & 78 & 61 & 84 & \\
\hline Time from miscarriage cycle (months) & $5.4 \pm 3.5(4(3-6))$ & $5.1 \pm 2.4(5(3-6))$ & $5.9 \pm 2.9(5(4-7))$ & 0.43 \\
\hline Fresh consecutive cycle & 28/78(35.9\%) & 29/61(47.5\%) & $40 / 84(47.6 \%)$ & 0.24 \\
\hline Frozen-thawed consecutive cycle & $50 / 78(64.1 \%)$ & $32 / 61(52.5 \%)$ & $44 / 84(52.4 \%)$ & 0.24 \\
\hline Peak Estradiol levels (fresh cycle; pmol/l) & $\begin{array}{l}4527.7 \pm 2706.9(4268(3047- \\
6472))\end{array}$ & $\begin{array}{l}5164.9 \pm 3639.4(4821(3528- \\
6599)\end{array}$ & $\begin{array}{l}4805.7 \pm 2706.1(3961(2042- \\
5623))\end{array}$ & 0.56 \\
\hline Grade A embryos (\%) & $27.1+37.7$ & $28.0+36.2$ & $31.3+36.1$ & 0.91 \\
\hline Grade B embryos (\%) & $47.6+43.3$ & $58.0+40.6$ & $45.2+38.9$ & 0.70 \\
\hline Grade C embryos (\%) & $6.3+18.8$ & $1.3+3.7$ & $10.1+22.8$ & 0.21 \\
\hline $\mathrm{N}$ of transferred embryos & $1.6 \pm 0.8(2(1-2))$ & $1.7 \pm 0.8(2(1-2))$ & $2.0 \pm 1.0(2(1-3))$ & 0.41 \\
\hline ET $(\mathrm{mm})$ at day of hCG/LH/P & $9.9 \pm 2.1(10(8-11))$ & $10.3 \pm 2.3(10(9-11))$ & $9.1 \pm 2.4(10(9-11))$ & $0.005^{\mathrm{b}}$ \\
\hline \multicolumn{5}{|c|}{ Comparison of ET between miscarriage cycle and consecutive cycle ${ }^{a}$} \\
\hline Difference at day of hCG/LH/P (mm) & $0.0 \pm 1.7(0(-1-1))$ & $0.2 \pm 2.2(0(-1-1))$ & $-1.0 \pm 2.4(-1(-2-0))$ & $0.002^{\mathrm{b}}$ \\
\hline$\%$ of change at day of hCG/LH/P & $-0.9 \pm 17.4(1(-8-10))$ & $3.5 \pm 21.7(0(-8-10))$ & $-8.2 \pm 22.3(-8(-25-3))$ & $0.001^{\mathrm{b}}$ \\
\hline $\begin{array}{l}\text { Decrease of }>2 \mathrm{~mm} \text { in ET at day of hCG/ } \\
\mathrm{LH} / \mathrm{P}\end{array}$ & $11 / 78(14.1 \%)^{c}$ & $4 / 61(6.6 \%)^{C}$ & 25/84(29.8\%) & $\begin{array}{l}< \\
0.001\end{array}$ \\
\hline \multicolumn{5}{|l|}{ Reproductive outcomes } \\
\hline Implantation rate & $32 / 105(30.5 \%)^{c}$ & 18/102 (17.7\%) & 16/144 (11.1\%) & $\begin{array}{l}< \\
0.001\end{array}$ \\
\hline Clinical pregnancy rate & $30 / 78(38.5 \%)^{C}$ & $17 / 61(27.9 \%)$ & 14/84 (16.7\%) & 0.008 \\
\hline Miscarriage rate & $12 / 30(40.0 \%)$ & $7 / 17(41.2 \%)$ & $5 / 14(35.7 \%)$ & 0.947 \\
\hline Live birth rate & 18/78 (23.1\%) & 10/61 (16.4\%) & 9/84 (10.7\%) & 0.107 \\
\hline
\end{tabular}

Data presented as mean $\pm \mathrm{SD}$ (Median (IQR)) or $\mathrm{n} / \mathrm{N}(\%)$

Note: $D \& C$ Dilation and curettage, $E T$ Endometrial thickness, $h C G / L H / P$ Day of human chorionic gonadotropin administration in fresh IVF cycles, luteinizing hormone surge or progesterone administration in frozen-thawed cycles

${ }^{a}$ Evaluated by one-way ANOVA and Kruskal-Wallis Test, showing significant difference in post hoc test between the conservative management and the D\&C groups

${ }^{\text {b} E v a l u a t e d ~ b y ~ o n e-w a y ~ A N O V A ~ a n d ~ K r u s k a l-W a l l i s ~ T e s t, ~ s h o w i n g ~ s i g n i f i c a n t ~ d i f f e r e n c e ~ i n ~ p o s t ~ h o c ~ t e s t ~ b e t w e e n ~ b o t h ~ c o n s e r v a t i v e ~ a n d ~ M i s o p r o s t o l ~ a n d ~ t h e ~}$ D\&C group

${ }^{C}$ Evaluated by post hoc Bonferroni test, showing significant difference compared to the D\&C groups

from the difference between the conservative management and the $\mathrm{D} \& \mathrm{C}$ group according to the post hoc analysis (post hoc Bonferroni test).

Although once a successful implantation occurred, there was no difference in miscarriage rates between the groups $(p=0.95)$. There was a trend towards a lower live birth rate in the $\mathrm{D} \& \mathrm{C}$ group compared with the conservative and Misoprostol management groups, though the difference did not reach statistical significance $(10.7 \%$ vs. $23.1 \%$ and 16.4 , respectively, $p=0.11$ ).

Table 3 Logistic regression analysis of parameters associated with endometrial thickness above $8 \mathrm{~mm}$ in the cycle subsequent to the miscarriage cycle

\begin{tabular}{|c|c|c|c|c|}
\hline Parameter & OR & Lower $95 \% \mathrm{Cl}$ & Upper $95 \% \mathrm{Cl}$ & $P$ value \\
\hline Miscarriage management & & & & 0.02 \\
\hline $\mathrm{D} \& \mathrm{C}$ & Referent & & & \\
\hline Conservative & 2.68 & 1.10 & 6.53 & 0.03 \\
\hline Misoprostol & 3.44 & 1.28 & 9.25 & 0.01 \\
\hline Past delivery & 2.40 & 1.08 & 5.32 & 0.03 \\
\hline ET at day of $\mathrm{hCG} / \mathrm{LH} / \mathrm{P}$ in the miscarriage cycle & 9.38 & 4.11 & 21.39 & $<0.001$ \\
\hline
\end{tabular}

Note: $D \& C$ Dilation and curettage, $E T$ Endometrial thickness, $h C G / L H / P$ Day of human chorionic gonadotropin administration in fresh IVF cycles, luteinizing hormone surge or progesterone administration in frozen-thawed cycles 
A logistic regression analysis was performed for the prediction of clinical pregnancy in the cycle subsequent to the miscarriage cycle. Both univariate and multivariate analyses found that the only significant independent parameters associated with clinical pregnancy were the mother's age at the following cycle and the miscarriage management approach (Conservative and Misoprostol compared to D\&C) (Table 4).

We further performed an additional analysis that included only patients in which the subsequent IVF cycle was of the same type (fresh or frozen-thawed) as the miscarriage cycle (Suppl. Table 1). In line with the analysis of the entire study population above (Tables $1 \& 2$ ), we observed significant differences in the average ET at the day of hCG/LH/P administration between the miscarriage management approaches. D\&C was associated with a greater average reduction in ET per patient at hCG/LH/P day in the subsequent cycle compared to conservative management and Misoprostol $(-0.9 \mathrm{~mm}$ vs. $0.0 \mathrm{~mm}$ and $0.5 \mathrm{~mm}$, respectively; $p=0.004$ ). Furthermore, management by $\mathrm{D} \& \mathrm{C}$ was the only approach associated with increased rates of ET thinning under 7 and $8 \mathrm{~mm}$ in the following cycle $(p=0.008$ and 0.035; respectively).

Implantation and clinical pregnancy were significantly lower in the subsequent IVF cycle following D\&C compared to conservative and Misoprostol management groups (Suppl. Table 1).

When comparison between the three management groups was performed separately for fresh and frozenthawed cycles, similar significant results were demonstrated for most parameters. The peak E2 levels in the fresh cycles were similar in the three sub-groups. A significant $(p<0.05)$ decline in ET following D\&C compared to similar ET after conservative management and Misoprostol was observed in both types of transfers. The difference in rates of a significant ET decrease (more than $2 \mathrm{~mm}$ ) following $\mathrm{D} \& \mathrm{C}$ compared with the two other approaches was statistically significant $(p<0.05$ for both types of cycles). In frozen-thawed cycles, clinical pregnancy rates were lowest in the $D \& C$ and highest in the conservative management group $(p<0.05)$. Although not significant, a similar trend was documented in the fresh transfer group (data available upon request).

\section{Discussion}

In this study, we analyzed the effect of three miscarriage management approaches following an IVF cycle - expectant management (conservative), medical treatment (Misoprostol) and D\&C, on endometrial thickness, implantation rates and pregnancy outcomes in the subsequent IVF attempt. To the best of our knowledge, this is the first study that compares ET at miscarriage IVF cycles with ET at subsequent IVF cycles with regard to the three miscarriage management approaches.

Our major finding was endometrial thinning in the subsequent IVF cycle following D\&C compared with the other miscarriage treatment approaches. A statistically significant thinning of average ET was observed after $D \& C$ in comparison to the other management methods. This was only observed in the $\mathrm{D} \& \mathrm{C}$ group by a significant increase in the prevalence of ET thinning by more than $2 \mathrm{~mm}$, and in the rate of patients who had ET decrease below 7 and $8 \mathrm{~mm}$ in the subsequent cycle.

Endometrial thinning after repeated D\&C was seen in several previous studies [19-21]. Shufaro et al. (2008) reported on a spectrum of post-curettage endometrial injuries ranging from a thin and unresponsive endometrium to Asherman syndrome [22]. Nevertheless, Moon et al., suggested that the decrease in ET after $\mathrm{D} \& \mathrm{C}$ is transient, lasts no longer than 6 months and have little or no clinical meaning [23]. A previous study by Tamir et al. examined 41 patients treated with D\&C and 32 patients treated with Misoprostol for spontaneous miscarriage following IVF cycles. They also showed a trend towards thinning of the endometrium after $\mathrm{D} \& \mathrm{C}$, though not significant $(10.4 \mathrm{~mm}$ before vs. $9.4 \mathrm{~mm}$ after D\&C; $p=0.06$ ). However, when comparing the two miscarriage management approaches, they reported a non-significant difference regarding ET on hCG administration day and similar pregnancy rates in subsequent cycles [24].

Our data suggested similar ET in subsequent IVF cycles after expectant management in comparison to medical treatment. Moreover, while following $\mathrm{D} \& \mathrm{C}$, the ET was significantly thinner at the time of hCG/LH/progesterone administration in the subsequent IVF cycle in comparison to the miscarriage IVF cycle, the ET was not significantly altered after expectant and Misoprostol managements. It

Table 4 Logistic regression analysis for prediction of clinical pregnancy in an In-vitro fertilization cycle following a miscarriage cycle

\begin{tabular}{llccc}
\hline Parameter & OR & Lower 95\% Cl & Upper 95\% Cl & \\
\hline Miscarriage management & & & & \\
D\&C & Referent & & 0.022 \\
Conservative & 2.68 & 1.34 & 6.10 & 0.01 \\
Misoprostol & 2.28 & 0.99 & 5.23 & 0.05 \\
Mother's age & 0.91 & 0.86 & 0.96 & $<0.001$ \\
\hline
\end{tabular}

Note: D\&C Dilation and curettage 
may be speculated that D\&C may be associated with a deleterious effect on stem cells in the basilar layer of the endometrium, resulting in a potential adverse effect on endometrial regeneration [19]. In contrast, both Misoprostol and expectant management are not associated with surgical trauma to the endometrium and give rise to expulsion of conception by other mechanisms. Misoprostol softens the cervix, primarily through an inflammatory response that leads to collagen degradation [25] and induces uterine contractions [26]. As for expectant management, the mechanism of miscarriage includes entry of maternal blood into the intervillous space that disrupts the maternal-embryonal interface [27].

There is inconsistent data in the literature regarding the optimal ET for embryonic implantation and successful pregnancy outcome after IVF. The lowest thickness under which poorer implantation rate has been reported is variable in different studies ranging from $6 \mathrm{~mm}$ to 10 $\mathrm{mm}$ [3-14]. The different methodologies in the reported studies such as the cycle day at which ET was measured and diverse measurement settings probably contribute to the literature inconsistency. A recent comprehensive study that analyzed 40,000 embryo transfer cycles suggested a decline in clinical pregnancy and live birth rates at ET below $8 \mathrm{~mm}$ in fresh embryo transfers and below $7 \mathrm{~mm}$ in frozen-thawed embryo transfers [28]. It has been suggested that the decrease in pregnancy outcomes is related to increased exposure to higher oxygen concentrations in the endometrial basal layer when the endometrium is thinner, which might be detrimental for embryo implantation [29].

In line with the literature, we showed a significant reduction in implantation and clinical pregnancy rates in the D\&C group, where the average ET was thinner, and the ET decrease below 7 and $8 \mathrm{~mm}$ in the subsequent cycle was significant. Although these findings were primarily due to the difference between the conservative and the D\&C groups, whereas the Misoprostol showed a lesser advantage in implantation and pregnancy rates over the $\mathrm{D} \& \mathrm{C}$ group, our logistic regression model revealed that both conservative management and Misoprostol treatment were positively associated with clinical pregnancy at the subsequent cycle compared to D\&C.

Our study was based on retrospective analysis of all patients that had a miscarriage after IVF treatment in our unit between 2006 and 2017. In an attempt to overcome, at least in part, the limitations of a retrospective study, we analyzed the ET before and after intervention, in the subsequent IVF cycle, per each patient to reduce interpatient variability that could mask the results. Except for previous deliveries and miscarriage week (which were highest in the D\&C group), there were no differences between the groups regarding patients' characteristics, gynecological history, cause of infertility and level of estradiol at hCG administration day. The differences between the study groups in the parameters of the miscarriage cycles regarding the number of previous deliveries and gestational age at miscarriage are limitation of the study. Nevertheless, linear and logistic regression analyses did not show that gestational week at miscarriage was correlated with ET, ET decrease below $8 \mathrm{~mm}$ or clinical pregnancy rates at the subsequent IVF cycle. Still, the gestational week at diagnosis could have an effect on the selection of the management modality by the patients. An additional limitation was the lack of data regarding the size of the gestational sac, the presence of an embryo and embryonal heartbeat loss in the miscarriage pregnancy. In contrast to a previous study that suggested a decrease in ET as a function of age [30]. A logistic regression analysis in our study did not find a correlation between patient's age and ET, though the multivariate analysis for clinical pregnancy did show a positive association with younger age. Nevertheless, the patients in the D\&C group were not older compared to the other study groups.

In conclusion, the results of the study suggest that $\mathrm{D} \& \mathrm{C}$ for miscarriage is associated with decreased ET and higher rates of thin endometrium below 7 and $8 \mathrm{~mm}$ in subsequent IVF cycles compared with expectant management and Misoprostol treatment. In addition, the implantation and clinical pregnancy rates of subsequent IVF cycles were significantly lower after $\mathrm{D} \& \mathrm{C}$, compared to conservative and medical managements of miscarriage Our data may assist in counseling patients confronted with making the choice of management of a miscarriage after IVF as well as after a spontaneous pregnancy.

\section{Supplementary Information}

The online version contains supplementary material available at https://doi. org/10.1186/s12958-021-00780-7.

Additional file 1: Table S1. Basic parameters, endometrium thickness (ET) and reproductive outcomes in the different management groups of consecutive similar type cycle to the miscarriage cycle.

\section{Acknowledgments}

None.

\section{Authors' contributions}

Gilad Karavani and Heli Alexandroni have contributed substantially to the conception and design of the study, analysis and interpretation of data and drafting the article. G. K and H. A have approved the final version of the study. Daniel Sheinin and Uri P Dior have contributed substantially to the acquisition, analysis and interpretation of data and drafting and revision of the article. D. S and U.P.D have approved the final version of the study. Alex Simon and Assaf Ben-Meir have contributed substantially to the conception and design of the study, analysis and interpretation of data and drafting and revision of the article. A. S and A.B.M have approved the final version of the study. Benjamin Reubinoff has contributed substantially to the conception and design of the study, acquisition, analysis and interpretation of data and drafting and revision of the article. B. R has approved the final version of the study.

Funding

No funding was received for this study. 


\section{Availability of data and materials}

The datasets used and/or analyzed during the current study are available from the corresponding author on reasonable request.

\section{Declarations}

\section{Ethics approval and consent to participate}

The study was approved by the Human Research Ethics Committees of the Hadassah University hospital (IRB 0657-17-HMO) and conforms to the provisions of the declaration of Helsinki.

\section{Consent for publication}

Not applicable.

\section{Competing interests}

The authors declare that they have no competing interests.

\section{Author details}

'Infertility and IVF Unit, Department of Obstetrics and Gynecology, Hadassah Ein-Kerem Medical Center and Faculty of Medicine, Hebrew University of Jerusalem, Jerusalem, Israel. ${ }^{2}$ Department of Obstetrics and Gynecology, Shaare Zedek Medical Center and Faculty of Medicine, Hebrew University of Jerusalem, Jerusalem, Israel. ${ }^{3}$ Faculty of Medicine, Hebrew University of Jerusalem, Jerusalem, Israel.

Received: 8 March 2021 Accepted: 10 June 2021

Published online: 22 June 2021

\section{References}

1. Kovacs P, Matyas S, Boda K, Kaali SG. The effect of endometrial thickness on IVF/ICSI outcome. Hum Reprod. 2003;18(11):2337-41. https://doi.org/10.1 093/humrep/deg461.

2. Zhao J, Zhang Q, Wang Y, Li Y. Endometrial pattern, thickness and growth in predicting pregnancy outcome following 3319 IVF cycle. Reprod BioMed Online. 2014;29(3):291-8. https://doi.org/10.1016/j.rbmo.2014.05.011.

3. Oliveira JB, Baruffi RL, Mauri AL, Petersen CG, Borges MC, Franco JG Jr. Endometrial ultrasonography as a predictor of pregnancy in an in-vitro fertilization programme after ovarian stimulation and gonadotrophinreleasing hormone and gonadotrophins. Hum Reprod. 1997;12(11):2515-8. https://doi.org/10.1093/humrep/12.11.2515.

4. Zhiqin B, Sun Y. The Impact of Endometrial Thickness on the Day of Human Chorionic Gonadotrophin (hCG) Administration on Ongoing Pregnancy Rate in Patients with Different Ovarian Response. PLoS One. 2015;10:e0145703.

5. Wu Y, Gao X, Lu X, Xi J, Jiang S, Sun Y, et al. Endometrial thickness affects the outcome of in vitro fertilization and embryo transfer in normal responders after GnRH antagonist administration. Reprod Biol Endocrinol. 2014;12:96.

6. Kumbak B, Erden HF, Tosun S, Akbas H, Ulug U, Bahçeci M. Outcome of assisted reproduction treatment in patients with endometrial thickness less than 7 mm. Reprod BioMed Online. 2009;18(1):79-84. https://doi.org/10.101 6/S1472-6483(10)60428-2

7. Kasius A, Smit JG, Torrance HL, Eijkemans MJ, Mol BW, Opmeer BC, et al. Endometrial thickness and pregnancy rates after IVF: a systematic review and meta-analysis. Hum Reprod Update. 2014;20(4):530-41. https://doi.org/1 0.1093/humupd/dmu011.

8. Al-Ghamdi A, Coskun S, Al-Hassan S, Al-Rejjal R, Awartani K. The correlation between endometrial thickness and outcome of in vitro fertilization and embryo transfer (IVF-ET) outcome. Reprod Biol Endocrinol. 2008;6(1):37-41. https://doi.org/10.1186/1477-7827-6-37.

9. Aydin T, Kara M, Turktekin N. Relationship between endometrial thickness and in vitro fertilization-intracytoplasmic sperm injection outcome. Int J Fertil Steril. 2013;7(1):29-34.

10. Chan JM, Sukumar Al, Ramalingam M, Ranbir Singh SS, Abdullah MF. The impact of endometrial thickness (EMT) on the day of human chorionic gonadotropin (hCG) administration on pregnancy outcomes: a 5-year retrospective cohort analysis in Malaysia. Fertil Res Pract. 2018;11:4-5.

11. Noyes N, Liu HC, Sultan K, Schattman G, Rosenwaks Z. Endometrial thickness appears to be a significant factor in embryo implantation in invitro fertilization. Hum Reprod. 1995;10(4):919-22. https://doi.org/10.1093/ oxfordjournals.humrep.a136061.
12. Rashidi BH, Sadeghi M, Jafarabadi M, Tehrani Nejad ES. Relationships between pregnancy rates following in vitro fertilization or intracytoplasmic sperm injection and endometrial thickness and pattern. Eur J Obstet Gynecol Reprod Biol. 2005;120(2):179-84. https://doi.org/10.1016/j.ejogrb.2 004.08.016.

13. Dickey RP, Olar TT, Curole DN, Taylor SN, Rye PH. Endometrial pattern and thickness associated with pregnancy outcome after assisted reproduction technologies. Hum Reprod. 1992;7(3):418-21. https://doi.org/10.1093/ oxfordjournals.humrep.a137661.

14. De Geyter C, Schmitter M, De Geyter M, Nieschlag E, Holzgreve W, Schneider HP. Prospective evaluation of the ultrasound appearance of the endometrium in a cohort of 1,186 infertile women. Fertil Steril. 2000;73(1): 106-13. https://doi.org/10.1016/S0015-0282(99)00484-7.

15. Weissman A, Gotlieb L, Casper RF. The detrimental effect of increased endometrial thickness on implantation and pregnancy rates and outcome in an in vitro fertilization program. Fertil Steril. 1999;71(1):147-9. https://doi. org/10.1016/S0015-0282(98)00413-0.

16. Sundstrom P. Establishment of a successful pregnancy following in-vitro fertilization with an endometrial thickness of no more than $4 \mathrm{~mm}$. Hum Reprod. 1998;13(6):1550-2. https://doi.org/10.1093/humrep/13.6.1550.

17. Quintero RB, Sharara Fl, Milki AA. Successful pregnancies in the setting of exaggerated endometrial thickness. Fertil Steril. 2004;82(1):215-7. https://doi. org/10.1016/j.fertnstert.2004.02.099.

18. Wang JX, Norman RJ, Wilcox AJ. Incidence of spontaneous abortion among pregnancies produced by assisted reproductive technology. Hum Reprod. 2004;19(2):272-7. https://doi.org/10.1093/humrep/deh078.

19. Azumaguchi A, Henmi H, Saito M, Itabashi M. Role of dilatation and curettage in the etiology of thin endometrium. Stockholm: Abstracts of the 27th Annual Meeting of ESHRE; 3 July - 6 July; 2011.

20. Davar R, Dehghani Firouzabadi R, Chaman AK. Dilatation and curettage effect on the endometrial thickness. Iran Red Crescent Med J. 2013;15(4): 350-5. https://doi.org/10.5812/ircmj.9863.

21. Netter AP, Boutaleb Y, Hallez JP, Minh HN, Bessis R, Lambert AE. Recurrent abortions: unpublished syndrome suggesting the explanation of fetal death. C R Acad Sci. 1996;319(7):637-8.

22. Shufaro $Y$, Simon A, Laufer N, Fatum M. Thin unresponsive endometrium - a possible complication of surgical curettage compromising ART outcome. J Assist Reprod Genet. 2008;25(8):421-5. https://doi.org/10.1007/s10815-008-9245-y.

23. Moon KS, Richter KS, Levy MJ, Widra EA. Does dilation and curettage versus expectant management for spontaneous abortion in patients undergoing in vitro fertilization affect subsequent endometrial development? Fertil Steril. 2009;92(5):1776-9. https://doi.org/10.1016/j.fertnstert.2009.05.045.

24. Tamir R, Allouche S, Weissman A, Haikin Herzberger E, Oberman-Farhi S, Shalom-Paz E, et al. The effect of medical versus surgical treatment of spontaneous miscarriage on subsequent in vitro fertilization cycles. Gynecol Endocrinol. 2016;32(3):231-3. https://doi.org/10.3109/09513590.2015.1110693.

25. Aronsson A, Ulfgren AK, Stabi B, Stavreus-Evers A, Gemzell-Danielsson K. The effect of orally and vaginally administered misoprostol on inflammatory mediators and cervical ripening during early pregnancy. Contraception. 2005;72(1):33-9. https://doi.org/10.1016/j.contraception.2005.02.012.

26. Tang OS, Gemzell-Danielsson K, Ho PC. Misoprostol: pharmacokinetic profiles, effects on the uterus and side-effects. Int J Gynaecol Obstet. 2007; 99:S160-7. https://doi.org/10.1016/j.ijgo.2007.09.004.

27. Schwärzler P, Holden D, Nielsen S, Hahlin M, Sladkevicius P, Bourne T. The conservative management of first trimester miscarriages and the use of colour Doppler sonography for patient selection. Hum Reprod. 1999;14(5): 1341-5. https://doi.org/10.1093/humrep/14.5.1341.

28. Liu KE, Hartman M, Hartman A, Luo ZC, Mahutte N. The impact of a thin endometrial lining on fresh and frozen-thaw IVF outcomes: an analysis of over 40000 embryo transfers. Hum Reprod. 2018;33(10):1883-8. https://doi. org/10.1093/humrep/dey281.

29. Casper RF. It's time to pay attention to the endometrium. Fertil Steril. 2011; 96(3):519-21. https://doi.org/10.1016/j.fertnstert.2011.07.1096.

30. Amir W, Micha B, Ariel H, Liat L-G, Jehoshua D, Adrian S. Predicting factors for endometrial thickness during treatment with assisted reproductive technology. Fertil Steril. 2007;87(4):799-804. https://doi.org/10.1016/j. fertnstert.2006.11.002.

\section{Publisher's Note}

Springer Nature remains neutral with regard to jurisdictional claims in published maps and institutional affiliations. 